\title{
Divergent predation environment between two sister species of livebearing fishes (Cyprinodontiformes: Poeciliidae) predicts boldness, activity, and exploration behavior
}

\author{
David A. Money ${ }^{1}$, Spencer J. Ingley ${ }^{1}$ \& Jerald B. Johnson ${ }^{1,2}$ \\ 1. Evolutionary Ecology Laboratories, Department of Biology, 4102 LSB, Brigham Young University, Provo, UT 84602, \\ USA; money.david260@gmail.com,sjingley@gmail.com \\ 2. Monte L. Bean Life Science Museum, Brigham Young University, Provo, UT 84602, USA; jerry.johnson@byu.edu
}

\author{
Received 20-IV-2016. C Corrected 26-VIII-2016. $\quad$ Accepted 28-IX-2016.
}

\begin{abstract}
Predators can influence a variety of prey traits, including behavior. Traits such as boldness, activity rate, and tendency to explore can all be shaped by predation risk. Our study examines the effects of predation on these behaviors by considering a natural system in which two sister species of livebearing fishes, Brachyrhaphis roseni and B. terrabensis, experience divergent predation environments. In February of 2013, we collected fish in the Río Chiriquí Nuevo drainage, Chiriquí, Panama, and conducted behavioral assays. Using open-field behavioral assays, we evaluated both juveniles and adults, and males and females, to determine if there were differences in behavior between ontogenetic stages or between sexes. We assessed boldness as 'time to emerge' from a shelter into a novel environment, and subsequently measured activity and exploration within that novel environment. We predicted that $B$. roseni (a species that co-occurs with predators) would be more bold, more active, and more prone to explore, than $B$. terrabensis (a species that does not co-occur with predators). In total, we tested 17 juveniles, 21 adult males, and 20 adult females of $B$. roseni, and 19 juveniles, 19 adult males, and 18 adult females of $B$. terrabensis. We collected all animals from streams in Chiriquí, Panama in February 2013, and tested them following a short acclimation period to laboratory conditions. As predicted, we found that predation environment was associated with several differences in behavior. Both adult and juvenile $B$. roseni were more active and more prone to explore than $B$. terrabensis. However, we found no differences in boldness in either adults or juveniles. We also found a significant interaction between 'sex' and 'species' as predictors of boldness and exploration, indicating that predation environment can affect behaviors of males and females differently in each species. Our work demonstrates the importance of considering sex and life history stage when evaluating the evolution of behavior. Rev. Biol. Trop. 65 (1): 267-277. Epub 2017 March 01.
\end{abstract}

Key words: activity, behavioral divergence, boldness, Brachyrhaphis, exploration, predation environment.

How environmental factors influence the evolution of behavior has long interested behavioral ecologists (Foster, 1999; Foster \& Endler, 1999). Predation environment [i.e., the presence or absence of predators, and associated environmental factors (Johnson \& Belk, 2001)] can be an important selective agent that acts on a variety of prey traits (Reznick \& Endler, 1982; Fraser \& Gilliam, 1987; Gale, Johnson, Schaalje, \& Belk, 2013), including behavior (Stamps, 2007). Behavior is thought to be an important target of selection because it directly affects predator-mediated mortality risk (Magnhagen, Hellstrom, Borcherding, \& Heynen, 2012; Ingley et al., 2014a). Furthermore, other environmental variables that correlate with the presence of predators might facilitate trade-offs that favor increased activity levels. For example, populations that experience high levels of predation often occur at lower densities (Kokko \& Rankin, 2006), which could favor individuals that are more bold and active in order to increase encounter rates with potential mates. In contrast, 
increased activity levels in prey might actually increase mortality risk (Brodin, 2009), even if increased activity rates did increase encounters with potential mates. Hence, comparative work evaluating how behavior evolves in populations that occur in divergent predation environments should aid our understanding of the adaptive nature of these behavioral traits, and help us better understand why a particular behavior might occur in some contexts but not in others. Particularly compelling are natural replicates -where consistent patterns of divergence occur in multiple independent lineages (Schluter, 2000)- because they allow for more robust conclusions about the underlying causes of trait evolution.

Stream fishes that occupy different predation environments are frequently used to understand how behavior evolves in different predation environments (Seghers, 1974; Magurran, Seghers, Carvalho, \& Shaw, 1992; Kelley \& Magurran, 2003; Archard \& Braithwaite, 2011a, b; Archard, Earley, Hanninen, \& Braithwaite, 2012). Among the best studied are livebearing fishes (Harris, Ramnarine, Smith, \& Pettersson, 2010; Smith \& Blumstein, 2010; Archard \& Braithwaite, 2011a, b), including species in the genus Brachyrhaphis (Archard \& Braithwaite, 2011a, b; Ingley et al., 2014a, b; Ingley, Rehm, \& Johnson, 2014). The presence or absence of predators is often correlated with a suite of environmental differences, such as the presence of potential competitors, resource availability, stream flow, and population densities (Johnson, 2002). Thus, in this paper we use 'predation environment' to describe a suite of environmental differences among populations. To date, much of the work in Brachyrhaphis has been done comparing populations within species, focusing on the role of predation environment in driving divergence in behavior (Archard et al., 2012; Ingley et al., 2014a), morphology (Wesner, Billman, Meier, \& Belk, 2011; Hassell, Meyers, Billman, Rasmussen, \& Belk, 2012; Ingley et al., 2014b), and life-history traits (Johnson, 2002; Johnson \& ZunigaVega, 2009; Billman, Rasmussen, Creighton, Johnson, \& Belk, 2014) among populations within species. For example, in separate studies, research on $B$. rhabdophora and on $B$. episcopi showed that populations within the same drainage that experienced divergent predation environments showed common patterns of life history evolution; moreover, these patterns were identical to those in other Poeciliids (Reznick \& Endler, 1982; Johnson, 2002; Riesch, Martin, \& Langerhans, 2013). In all cases, individuals from high-predation environments matured more quickly and at a smaller size, and had more and smaller offspring than those from low-predation environments. This focus on within species divergence in Brachyrhaphis has provided excellent insight into the repeatability of evolution and the role of predation in driving trait divergence (Ingley \& Johnson, 2016a, b). However, given that these studies have focused on recently diverged population pairs within a species, we still know very little about how trait diversification is maintained post-speciation.

Our study takes advantage of a unique sister species pair within Brachyrhaphis $-B$. roseni and $B$. terrabensis - in order to explore how behavioral trait divergence is maintained once speciation is complete, and if these differences are consistent across different age classes (e.g., juveniles vs. adults). These species are sister species (Ingley, Reina, Bermingham, \& Johnson, 2015) that occur in the same river drainages throughout Northwestern Panama and Southeastern Costa Rica, but each occupies a different predation environment. Brachyrhaphis roseni lives in high-predation environments that contain abundant ambush predators (e.g., Hoplias microlepis and Gobiomorus dormitor), have higher resource availability, and lower population densities than the low-predation environments where $B$. terrabensis occurs (Ingley et al., 2014a, b; Ingley \& Johnson, 2016a, b). Therefore, using B. roseni and $B$. terrabensis as a model system, we are able to test whether patterns of behavioral divergence that have been documented within species (Brown \& Braithwaite, 2004; Brown, Jones, \& Braithwaite, 2007; Archard \& Braithwaite, 2011a, b), are also present between 
species. This work has the potential to provide insight into how behavioral trait variation is maintained post-speciation.

Although several studies have considered the question of whether predation environment affects behavior in Brachyrhaphis (e.g., Archard \& Braithwaite, 2011a; Ingley et al., 2014a), our study builds on previous work by comparing behaviors among species for both juveniles and adults -thus allowing us to determine how predation environment affects behavior during different stages of ontogenetic development- and by comparing males with females to test for sex differences. Previous work has focused on adults (Archard \& Braithwaite, 2011b; Ingley et al., 2014a), thus ignoring the potential for different size classes to experience different selective pressures (Johnson \& Zuñiga-Vega, 2009; Ingley \& Johnson, 2016b). Furthermore, previous work in a closely related species (B. episcopi) found that boldness behavior varied with size (Brown \& Braithwaite, 2004). Hence, we anticipate that differences in adaptive behaviors could vary by age. By comparing males and females we can determine if sex-dependent behavior differs between species. Male poeciliid fishes, including male Brachyraphis, prefer mating with multiple partners to increase their reproductive chances (Simcox, Colegrave, Heenan, Howard, $\&$ Braithwaite, 2005). However, females are able to store sperm, and need not expend energy finding partners with which to mate. Therefore, males that are more active and explorative should increase their chances of finding unique potential mates, and we anticipate that behavior could vary by sex.

Hence, the objectives of our study were twofold. First, we tested for differences in boldness, activity, and exploration levels between $B$. roseni and B. terrabensis. We compared adults and juveniles of each species separately, and also compared adults to juveniles for each group to determine if behavior was consistent across different age classes. We hypothesized that, overall, $B$. roseni would be bolder than B. terrabensis, a behavior potentially selected for by the presence of ambush predators in B. roseni but not in B. terrabensis. We also predicted that $B$. rosen $i$ would be more active and more likely to explore than $B$. terrabensis, given that these traits would help $B$. roseni maximize growth rates and reach maturity faster in the presence of predators (Roff, 1992). Finally, we predicted that juveniles and adults would not differ, potentially reflecting the presence of 'personality' in these species (i.e., individual differences in behavior that are consistent over time). Second, we tested for differences in these same behavioral traits between sexes within each species. We predicted that males in both species should be more active and more bold than females, given that males that aggressively court females and increase their activity rates garner increased mating opportunities.

\section{METHODS AND MATERIALS}

Live fish collection and care: We used hand held seines to collect live $B$. roseni and B. terrabensis from separate streams in the Rio Chiriquí Nuevo drainage in Chiriquí, Panama in February 2013. We collected B. roseni from a low-elevation site $\left(08^{\circ} 25^{\prime} 29^{\prime \prime} \mathrm{N}-82^{\circ} 24^{\prime} 57^{\prime}\right.$ ' $\mathrm{W}$, elevation: 18 meters), and B. terrabensis from a high-elevation site $\left(08^{\circ} 45^{\prime} 56^{\prime \prime} \mathrm{N}\right.$ 82²6'01" W, elevation: 1073 meters). Upon collection, we immediately transported fish from each population to separate 300 liter pools housed in David, Panama where they were held for at least seven days prior to testing in order to acclimate fish to holding conditions. We collected and tested B. roseni first, followed by $B$. terrabensis one week later. Fish were held at densities of approximately 80 fish per pool and were fed TetraColor Tropical Flakes twice daily, including the day of testing. Fish were kept outdoors under natural light ( 12L: 12D) and ambient temperature conditions $\left(24-26^{\circ} \mathrm{C}\right)$, and each pool contained three large stones to provide shelter.

Experimental assays for boldness, exploration and activity: In order to test for differences between predation environments, 
we conducted a two part behavioral assay that examined boldness, exploration, and activity levels for each individual. In order to be able to compare our results to other behavioral studies, we used previously established methods for measuring behavioral traits in live-bearing fishes (Archard \& Braithwaite, 2011b; Archard et al., 2012; Ingley et al., 2014a). These tests were conducted in an experimental arena that consisted of a $63.5 \times 41.5 \times 26 \mathrm{~cm}$ tank attached to a separate acclimation tank measuring 31 x $20.5 \times 26 \mathrm{~cm}$. The acclimation tank was connected to the test arena by a $14.5 \times 16 \mathrm{~cm}$ opaque cylinder. We visually divided the test arena (as viewed from above) into six equal sized zones along the long axis of the tank. A predetermined set of three of these zones contained a source of cover taken from the natural environment (e.g., rock or large decomposing leaf). The placement of these sources of cover were rearranged to increase novelty between replicate trials for a given individual, but maintained constant across individuals. For all trials, tanks were filled to $13 \mathrm{~cm}$ of water. We video recorded each trial using a Logitech C260 webcam that was mounted above the test arena. The day prior to testing, we sorted fish into approximately equal number of juveniles, males, and females of each species into separate four-liter holding tanks. For each trial, we carefully transferred a fish from its respective holding tank into the acclimation chamber where it was held for a two-minute acclimation period. An opaque plastic barrier prevented the fish from leaving the acclimation chamber prior to the start of the trial. This barrier was removed remotely via pulley so as to not startle the test individual once the trial was started. To assay boldness, we first measured the time to emerge from the acclimation chamber into the novel environment. We then gave each individual eight minutes to explore this environment. Following the initial trial, we moved the test fish to separate, individually occupied and marked tanks (4 liters each) in order to track their identity for the second replicate of the trial. Although it is possible that housing fish individually vs. collectively could have affected their behavior, we saw no evidence of this, and found no qualitative differences in our results when we used behavioral data from the first trial vs. the average of the two trials. Following a 24 hour wait period in individual tanks, we repeated the behavioral assay with rearranged refugia to enhance novelty during the second trial. At the conclusion of the second trial, we removed the subject from the test arena, euthanized with an overdose MS-222, and took standard length (SL) and mass measurements. All specimens were preserved in ethanol and deposited into the Monte L. Bean Life Science Museum for future work.

After the completion of the behavioral assays, we analyzed the digital recordings of each trial using Etholog v2.2.5 (Ottoni, 2000) to quantify fish activity and exploration levels. A single observer analyzed all of the videos to ensure consistency. From each trial we extracted four behavioral variables as indicators of our three focal behaviors. As a measure of boldness we scored time to emerge from the acclimation chamber (i.e., once an individual's entire body had left the acclimation chamber) into the novel environment and proportion of time spent in a zone with a shelter object; as a measure of activity we scored total proportion of time spent in motion; and as a measure of exploration we scored the rate of movement between the six zones (i.e., the number of zone boundaries crossed during the trial period). In total, we tested 17 juveniles, 21 adult males, and 20 adult females of $B$. roseni, and 19 juveniles, 19 adult males, and 18 adult females of B. terrabensis. All collecting and experiments were reviewed and approved by the Smithsonian Tropical Research Institute and Brigham Young University IACUC committees.

To test for differences in behavior between juveniles and adults from each of the two focal species, and to test for differences between sexes, we used an analysis of covariance framework (ANCOVA). This required two data transformations to meet the assumptions of the statistical model. We log transformed 'time to emerge' and 'SL' and then used these transformed values in each of the subsequent 
analyses. To test for differences in behavior between juveniles, we conducted separate ANCOVAs with each of the four behavioral measures as response variables, species as a main factor, and log SL as a covariate. Interaction terms between species and log SL were also included to control for size effects. To test for differences in behavior between adults, we again conducted separate ANCOVAs with each of the four behavioral measures as response variables, species and sex as main factors, and $\log$ SL as a covariate. Two and three way interactions between sex, species, and log SL were also included. The sex by species interaction allowed us to test if sexes responded to predation environments differently. We also conducted ANOVAs to test for a difference between age classes (i.e., adults vs. juveniles). For each behavioral measure, we generated a model with age and species as main effects, an interaction between age and species, and log $\mathrm{SL}$ as a covariate. Using log SL as a covariate in all of our ANCOVA models allowed us to control for any effect of size on each behavioral measure, as B. roseni and B. terrabensis significantly differ in both SL $\left(F_{1,141}\right.$ $=8.516, \mathrm{P}=0.004)$ and mass $\left(F_{1,141}=11.45, \mathrm{P}\right.$ $<0.001)$. Finally, we used ANOVA to test for differences in SL between species and sexes. All analyses were conducted in $\mathrm{R}$ ( $\mathrm{R}$ Core development team, 2008).

\section{RESULTS}

We found several significant differences in behavior between adult $B$. roseni and $B$. terrabensis. Interestingly, we did not find a difference between the two focal species in time to emerge (male B. roseni: $137.4 \pm 27.7$; female B. roseni: $131.5 \pm 25.7$; male B. terrabensis: $83.5 \pm 22.6$; female B. terrabensis: $322.3 \pm$ 47.4; Table 1; Fig. 1A), which has previously been found to differ both between these species and between other populations of poeciliid fish that occur in different predation environments. However, we did find a significant difference
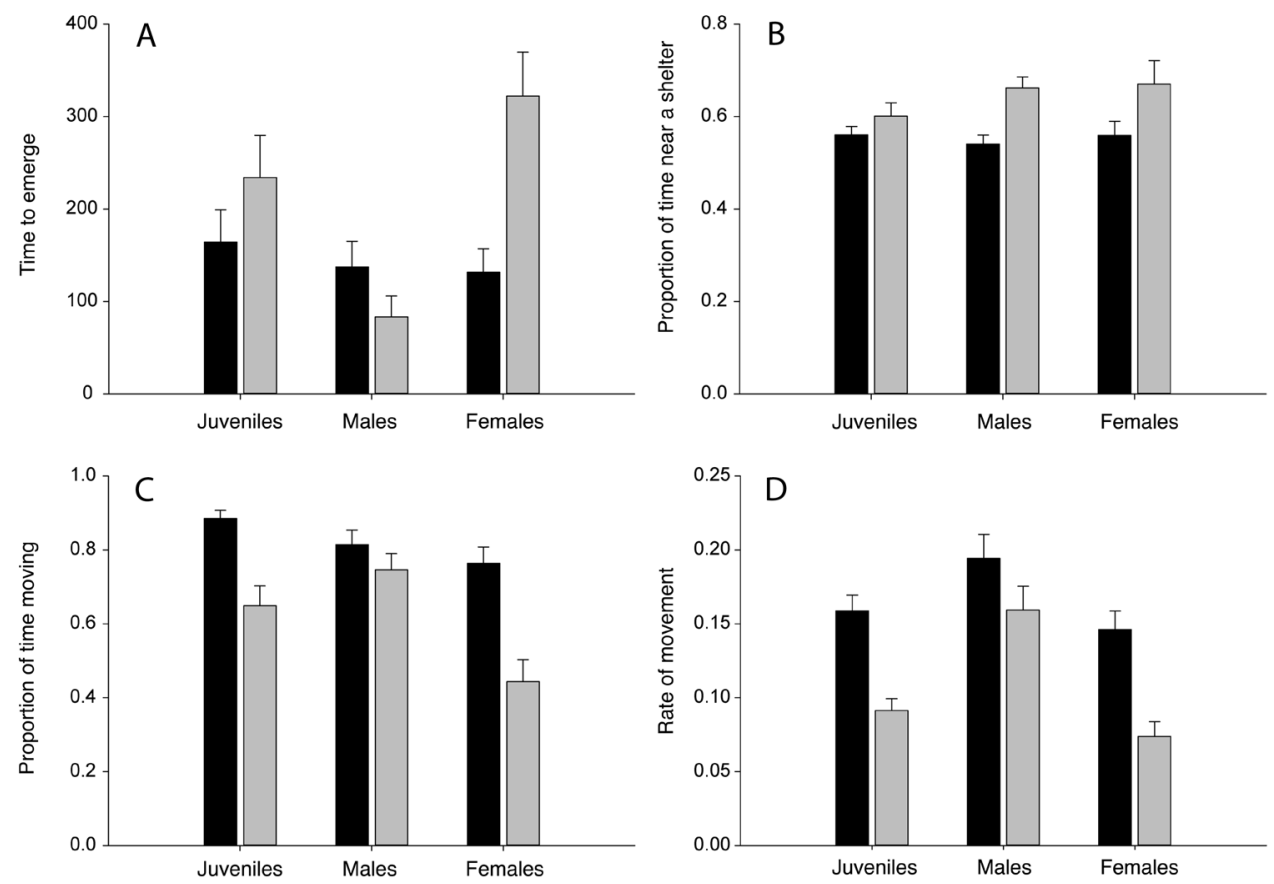

Fig. 1. Means ( \pm standard error) for: A) time to emerge (seconds); B) proportion of time near a shelter; C) proportion of time moving; and D) rate of movement. Brachyrhaphis roseni is presented in black and B. terrabensis is presented in gray. 
TABLE 1

ANCOVA table for model with adults

\begin{tabular}{|c|c|c|c|c|c|}
\hline Effect & Df & Sum Sq & Mean Sq & F-value & $P$ \\
\hline \multicolumn{6}{|l|}{ Time to Emerge } \\
\hline Species & 1 & 0.127 & 0.127 & 0.089 & 0.766 \\
\hline Sex & 1 & 23.361 & 23.361 & 16.324 & $<0.001$ \\
\hline $\log \mathrm{SL}$ & 1 & 0.150 & 0.150 & 0.105 & 0.747 \\
\hline Species by Sex & 1 & 23.959 & 23.959 & 16.742 & $<0.001$ \\
\hline Species by Log SL & 1 & 2.894 & 2.894 & 2.022 & 0.159 \\
\hline Sex by Log SL & 1 & 0.252 & 0.252 & 0.176 & 0.676 \\
\hline Species by Sex by Log SL & 1 & 0.190 & 0.190 & 0.133 & 0.716 \\
\hline \multicolumn{6}{|c|}{ Proportion of Time Near Shelter } \\
\hline Species & 1 & 0.335 & 0.335 & 13.209 & $<0.001$ \\
\hline Sex & 1 & 0.002 & 0.002 & 0.094 & 0.760 \\
\hline Log SL & 1 & 0.007 & 0.007 & 0.263 & 0.609 \\
\hline Species by Sex & 1 & 0.001 & 0.001 & 0.023 & 0.880 \\
\hline Species by Log SL & 1 & 0.000 & 0.000 & 0.019 & 0.890 \\
\hline Sex by Log SL & 1 & 0.001 & 0.001 & 0.028 & 0.867 \\
\hline Species by Sex by Log SL & 1 & 0.011 & 0.011 & 0.448 & 0.505 \\
\hline \multicolumn{6}{|l|}{ Proportion of Time Moving } \\
\hline Species & 1 & 0.733 & 0.733 & 13.484 & $<0.001$ \\
\hline Sex & 1 & 0.726 & 0.726 & 13.352 & $<0.001$ \\
\hline Log SL & 1 & 0.011 & 0.011 & 0.199 & 0.657 \\
\hline Species by Sex & 1 & 0.332 & 0.332 & 6.111 & 0.015 \\
\hline Species by Log SL & 1 & 0.046 & 0.046 & 0.837 & 0.363 \\
\hline Sex by Log SL & 1 & 0.004 & 0.003 & 0.064 & 0.802 \\
\hline Species by Sex by Log SL & 1 & 0.093 & 0.093 & 1.714 & 0.194 \\
\hline \multicolumn{6}{|l|}{ Rate of Movement } \\
\hline Species & 1 & 0.075 & 0.075 & 19.688 & $<0.001$ \\
\hline Sex & 1 & 0.083 & 0.083 & 21.622 & $<0.001$ \\
\hline $\log \mathrm{SL}$ & 1 & 0.002 & 0.002 & 0.622 & 0.432 \\
\hline Species by Sex & 1 & 0.004 & 0.004 & 1.104 & 0.296 \\
\hline Species by Log SL & 1 & 0.001 & 0.001 & 0.331 & 0.566 \\
\hline Sex by Log SL & 1 & 0.003 & 0.003 & 0.861 & 0.356 \\
\hline Species by Sex by Log SL & 1 & 0.006 & 0.006 & 1.662 & 0.201 \\
\hline
\end{tabular}

between species in time spent near a shelter. Specifically, B. terrabensis (males: $0.662 \pm$ 0.023; females: $0.669 \pm 0.051$ ) spent a significantly higher proportion of the trial near a shelter object (e.g., stone, leaf) than B. roseni (males: $0.541 \pm 0.019$; females: $0.559 \pm 0.030$; Table 1; Fig. 1B). We also found that species differed in the proportion of time moving and in the rate of movement, with $B$. roseni being more active and more prone to explore than $B$. terrabensis (see below for means $\pm \mathrm{SE}$; Table 1; Fig. 1C and Fig. 1D).
Juvenile B. roseni and B. terrabensis did not differ from each other in time to emerge from a shelter into a novel environment (164.3 \pm 35.1 and $234.2 \pm 45.6$, respectively) or in time spent near a shelter $(0.561 \pm 0.018$ and $0.601 \pm 0.029$, respectively; Table 2; Fig. 1A and Fig. 1B). Juvenile B. roseni spent a higher proportion of time moving than $B$. terrabensis $(0.885 \pm 0.023$ and $0.649 \pm 0.054$, respectively; Table 2; Fig. 1C), and more actively explored the arena $(0.159 \pm 0.011$ and $0.091 \pm 0.008$, respectively; Table 2; Fig. 1D). When testing 
TABLE 2

ANCOVA table for model with juveniles only

\begin{tabular}{|c|c|c|c|c|c|}
\hline Effect & Df & Sum Sq & Mean Sq & F-value & $\mathrm{P}$ \\
\hline \multicolumn{6}{|l|}{ Time to Emerge } \\
\hline Species & 1 & 0.023 & 0.023 & 0.012 & 0.912 \\
\hline SL & 1 & 0.305 & 0.305 & 0.162 & 0.689 \\
\hline Species by SL & 1 & 3.047 & 3.047 & 1.622 & 0.209 \\
\hline \multicolumn{6}{|c|}{ Proportion of Time Near Shelter } \\
\hline Species & 1 & 0.018 & 0.018 & 1.087 & 0.303 \\
\hline SL & 1 & 0.001 & 0.001 & 0.035 & 0.855 \\
\hline Species by SL & 1 & 0.013 & 0.013 & 0.791 & 0.379 \\
\hline \multicolumn{6}{|c|}{ Proportion of Time Moving } \\
\hline Species & 1 & 0.610 & 0.610 & 13.274 & $<0.001$ \\
\hline SL & 1 & 0.333 & 0.333 & 7.245 & 0.010 \\
\hline Species by SL & 1 & 0.093 & 0.093 & 2.019 & 0.163 \\
\hline \multicolumn{6}{|l|}{ Rate of Movement } \\
\hline Species & 1 & 0.049 & 0.049 & 26.345 & $<0.001$ \\
\hline SL & 1 & 0.002 & 0.002 & 1.2724 & 0.266 \\
\hline Species by SL & 1 & 0.00008 & 0.00008 & 0.0431 & 0.837 \\
\hline
\end{tabular}

for overall differences between age classes (i.e., juvenile vs. adult), we found no differences in any of our measured traits (all $\mathrm{P}>0.09$ ). We also found that this pattern was consistent across species (non-significant species by age interaction; all $\mathrm{P}>0.05$ ), indicating that juveniles and adults were equally similar to each other in each species.

Regarding sex differences, we found that males emerged more quickly from shelters than females (see above for means $\pm \mathrm{SE}$; Table 1 ; Fig. 1A). However, males did not differ from females in the proportion of time spent near a shelter (see above for means \pm SE; Fig. 1B). Males spent a higher proportion of time moving (B. roseni: $0.814 \pm 0.040 ;$ B. terrabensis: $0.746 \pm 0.044)$ than females (B. roseni: 0.764 \pm 0.044 ; B. terrabensis: $0.444 \pm 0.059)$. Males (B. roseni: $0.194 \pm 0.016$; B. terrabensis: 0.159 $\pm 0.016)$ also had a higher rate of movement than females $(B$. roseni: $0.146 \pm 0.012 ; B$. terrabensis: $0.074 \pm 0.010$; Table 1 ; Fig. $1 \mathrm{C}$ and Fig. 1D). We found evidence for a significant interaction between species and sex for both 'time to emerge' and 'proportion of time moving' (Table 1; Fig. 1A and Fig. 1C). In both cases, this significant species by sex interaction appeared to be due to more exaggerated differences between females of each species than between males of each species.

\section{DISCUSSION}

Consistent with our predictions, we found that $B$. rosen $i$ was more bold, active and explorative than $B$. terrabensis. Our work was also consistent with previous work evaluating these traits in other species where comparisons were among populations that occur in different predation environments (Archard \& Braithwaite, 2011a, b; Ingley et al., 2014a). We also found that, overall; males expressed higher boldness, activity, and exploration levels than females, as predicted. However, our work reveals that important differences in behavioral divergence exist between juveniles and adults of the two species, and that between species sexual dimorphism of behavior was different.

Brachyrhaphis roseni and B. terrabensis behave differently for some behaviors, but not others. We found no difference in boldness 
between species for either juveniles or adults. We also found no evidence for differences between age classes in this behavior. However, the trend was that $B$. roseni tended to emerge more quickly than $B$. terrabensis, a pattern consistent with previous work on other poeciliids in general, and in these species specifically (Brown, Jones, \& Braithwaite, 2005; Dzieweczynski \& Crovo, 2011; Magnhagen et al., 2012; Ingley et al., 2014a, b). We did find that adult $B$. roseni spent significantly more time in exposed areas of the arena and away from possible refugia than did adult $B$. terrabensis, which might reflect anti-predator behavioral strategies. Given that the most common predators with which $B$. roseni occurs are ambush predators (e.g., Hoplias microlepis), spending more time in exposed areas could be selected for in the presence of predators that strike from less exposed areas. Interestingly, no such differences were found in juveniles, suggesting that this behavior might vary across ontogeny. This finding is not surprising given that there are no differences in juvenile mortality risk between predation environments (Ingley \& Johnson, 2016b). However, our test comparing adults to juveniles revealed no significant difference in this behavior, suggesting that it may in fact be fixed across different life stages, a pattern potentially indicative of 'personality.'

Brachyrhaphis roseni and B. terrabensis also differed in activity levels and exploration behavior. Importantly, this pattern was consistent in both juveniles and adults, but no differences were found between juveniles and adults within each species. Adult B. roseni were more active and more prone to explore than $B$. terrabensis, a pattern consistent with previous work with these species (Ingley et al., 2014a) and other poeciliid fishes (Archard \& Braithwaite, 2011a). By examining activity levels in juveniles, our work expands upon previous research. We found that juveniles follow a similar pattern to that observed in adults, with $B$. roseni more active than $B$. terrabensis. These differences in activity and exploration levels could be explained by differences in population density. Low population densities that reduce encounter rates between potential mates could favor increased activity levels to ensure sufficient opportunities to reproduce. This explanation has previously been invoked to explain increased activity levels in these taxa (Ingley et al., 2014a), but would not apply to non-reproductive juveniles. Alternatively, activity levels of populations occurring in different predation environments could be influenced by resource availability. In this system, resources are typically more abundant in highpredation streams relative to low-predation streams (Johnson, 2002). Populations with greater resource availability have been shown to have higher activity and exploration levels (Rowe, Pierson, \& McGraw, 2015). Differences in resource availability could affect behavior in both adults and juveniles, and could explain why we detected increased activity levels in fish from high-predation populations in both adults and juveniles. Therefore, it is possible that either different selective forces are acting on juveniles vs. adults (i.e., resource availability vs. mate availability, respectively), or that selection acting on either juveniles or adults favors stability of activity and exploration levels across ontogeny. Future work should evaluate ontogenetic stage-specific selection on behavioral traits to tease apart the underlying factors driving this pattern, or alternatively, if behavior is constrained across ontogeny despite potential difference in selection at different life history stages. Furthermore, given that we examined wild-caught individuals in this study, these differences could be the result of environmentally driven plasticity, or due to some interaction between genes and the environment. Future work would benefit from testing the genetic basis of these behaviors, which would provide crucial information regarding whether these differences are evolved or the result of plasticity.

The final objective of our study was to test for differences in behavior between sexes, including whether or not sex-specific behaviors varied between species from different predation environments. We found male and female behavior to be more similar in Brachyrhaphis 
roseni. In contrast, male and female behavior in B. terrabensis differed for boldness, activity, and exploration levels. Male B. terrabensis were more bold, active, and prone to explore than females. One explanation is that Brachyrhaphis roseni occur in high-predation environments, which might overshadow other selective forces. In contrast, the low-predation environment in which $B$. terrabensis occurs could allow other selective agents to shape differences between sexes in behavior. For example, male $B$. terrabensis were much more active than females, possibly due to fitness advantages based on mating patterns. However, female $B$. roseni might have also adapted to have higher boldness, activity, and exploration levels, since predation mortality risk is such a strong selective force, thus resulting in similar behavior across sex. Controlled experiments could help tease out this possibility.

Our study adds additional support to a growing body of work that suggests that behavior - particularly boldness, activity, and exploration levels- differs between populations or species that occur in divergent predation environments, and that these differences are consistent across different life history stages. By demonstrating differences in behaviors between species, and between sexes, we also show that behavioral diversification can be fine-tuned, consistent with the evolutionary challenges faced by organisms throughout their life.

\section{ACKNOWLEDGMENTS}

This work was funded by the Kennedy Center for International Studies at Brigham Young University and the BYU Department of Biology. Additional research support came from a BYU ORCA research grant to DM and from the National Geographic Young Explorers Grant to SJI. A U.S. National Science Foundation Graduate Research Fellowship supported SJI. We thank J. Rehm for help in the lab and in the field. The Smithsonian Tropical Research Institute provided help with permits and provided IACUC oversight.

\section{RESUMEN}

Ambientes de depredación divergente para dos especies hermanas de peces vivíparos (Cyprinodontiformes: Poeciliidae) predicen el comportamiento de audacia, actividad y exploración. Los depredadores pueden influenciar una variedad de rasgos de la presa, incluyendo el comportamiento. Los rasgos tales como la audacia, nivel de actividad, y la tendencia a explorar; pueden ser moldeados por el riesgo de depredación. Nuestro estudio examina los efectos de la depredación en el comportamiento al considerar un sistema natural en el que dos especies hermanas de peces vivíparos, Brachyrhaphis roseni y B. terrabensis, se presentan en ambientes de depredación divergente. En febrero 2013, recolectamos peces en el drenaje del Río Chiriquí Nuevo, Chiriquí, Panamá y llevamos a cabo ensayos de comportamiento. Al usar ensayos de comportamiento en campo abierto, se evaluó el comportamiento en juveniles y adultos, machos y hembras, para determinar si los patrones de divergencia diferían entre las etapas ontogenéticas o entre sexos. Se evaluó la audacia como "tiempo en salir" de un refugio a un ambiente nuevo, y posteriormente se midió la actividad y la exploración dentro de ese nuevo ambiente. Nosotros predijimos que $B$. roseni (una especie que se presenta con los depredadores) sería más audaz, activa y propensa a explorar que $B$. terrabensis (una especie que no se presenta con los depredadores). En total, probamos 17 jóvenes, 21 machos adultos, y 20 hembras adultas de B. roseni, y 19 jóvenes, 19 machos adultos, y 18 hembras adultas de $B$. terrabensis. Recogimos todos los animales en Chiriquí, Panamá en Febrero 2013, y los probamos después de un corto período de aclimatación a las condiciones de laboratorio. Como se predijo, se encontró que la depredación ambiental se asoció con varias diferencias en el comportamiento. Tanto adultos y jóvenes de $B$. roseni eran más activos y más propensos a explorar que $B$. terrabensis. Sin embargo, no se encontraron diferencias en la audacia en adultos o menores. También se encontró una interacción significativa entre "sexo" y "especie" como predictores de la audacia y la exploración, lo que indica que la depredación puede afectar el comportamiento de los machos y hembras de manera diferente en cada especie. Nuestro estudio demuestra la importancia de considerar el sexo y la etapa del ciclo de vida al evaluar la evolución del comportamiento.

Palabras clave: actividad, audacia, Brachyrhaphis, depredación ambiental, divergencia en el comportamiento, exploración.

\section{REFERENCES}

Archard, G. A., \& Braithwaite, V. A. (2011a). Increased exposure to predators increases both exploration and activity level in Brachyrhaphis episcopi. Journal of Fish Biology, 78(2), 593-601. doi:10.1111/j.1095-8649.2010.02880.x 
Archard, G. A., \& Braithwaite, V. A. (2011b). Variation in aggressive behaviour in the poeciliid fish $\mathrm{Bra}$ chyrhaphis episcopi: population and sex differences. Behavioural Processes, 86(1), 52-57.

Archard, G. A., Earley, R. L., Hanninen, A. F., \& Braithwaite, V. A. (2012). Correlated behaviour and stress physiology in fish exposed to different levels of predation pressure. Functional Ecology, 26(3), 637-645. doi:10.1111/j.1365-2435.2012.01968.x

Billman, E. J., Rasmussen, J. E., Creighton, J. C., Johnson, J. B., \& Belk, M. C. (2014). A multivariate approach to the analysis of within lifetime variation in life history. Methods in Ecology and Evolution, 5(8), $797-$ 805. doi:10.1111/2041-210x.12211

Brodin, T. (2009). Behavioral syndrome over the boundaries of life-carryovers from larvae to adult damselfly. Behavioral Ecology, 20, 30-37. doi: 10.1093/beheco/ arn111

Brown, C., \& Braithwaite, V. A. (2004). Size matters: a test of boldness in eight populations of the poeciliid Brachyraphis episcopi. Animal Behaviour, 68(6), 1325-1329.

Brown, C., Jones, F., \& Braithwaite, V. (2005). In situ examination of boldness-shyness traits in the tropical poeciliid, Brachyraphis episcopi. Animal Behaviour, 70, 1003-1009. doi:10.1016/j.anbehav.2004.12.022

Brown, C., Jones, F., \& Braithwaite, V. (2007). Correlation between boldness and body mass in natural populations of the poeciliid Brachyrhaphis episcopi. Journal of Fish Biology, 71(6), 1590-1601.

Dzieweczynski, T. L., \& Crovo, J. A. (2011). Shyness and boldness differences across contexts in juvenile threespined stickleback Gasterosteus aculeatus from an anadromous population. Journal of Fish Biology, 79(3), 776-788. doi:10.1111/j.1095-8649.2011.03064.x

Foster, S. A. (1999). The geography of behaviour: an evolutionary perspective. Trends in Ecology \& Evolution, 14(5), 190-195.

Foster, S. A., \& Endler, J. A. (1999). Thoughts on geographic variation in behavior. In S. A. Foster, \& J. A. Endler (Eds.), Geographic variation in behavior (pp. 287-305). New York: Oxford University Press.

Fraser, D. F., \& Gilliam, J. F. (1987). Feeding under predation hazard-response of the guppy and hart rivulus from sites with contrasting predation hazard. Behavioral Ecology and Sociobiology, 21(4), 203-209. doi:10.1007/bf00292500

Gale, B. H., Johnson, J. B., Bruce Schaalje, G., \& Belk, M. C. (2013). Effects of predation environment and food availability on somatic growth in the Livebearing Fish Brachyrhaphis rhabdophora (Pisces: Poeciliidae). Ecology and Evolution, 3(2), 326-333. doi:10.1002/ece3.459
Harris, S., Ramnarine, I. W., Smith, H. G., \& Pettersson, L. B. (2010). Picking personalities apart: estimating the influence of predation, sex and body size on boldness in the guppy Poecilia reticulata. Oikos, 119(11), 1711-1718.

Hassell, E. M. A., Meyers, P. J., Billman, E. J., Rasmussen, J. E., \& Belk, M. C. (2012). Ontogeny and sex alter the effect of predation on body shape in a livebearing fish: sexual dimorphism, parallelism, and costs of reproduction. Ecology and Evolution, 2(7), 17381746. doi:10.1002/ece3.278

Ingley, S. J., Rehm, J., \& Johnson, J. B. (2014). Size doesn't matter, sex does: a test for boldness in sister species of Brachyrhaphis fishes. Ecology and Evolution, 4(22), 4361-4369. doi:10.1002/ece3.1304

Ingley, S., Billman, E., Hancock, C., \& Johnson, J. (2014a). Repeated geographic divergence in behavior: a case study employing phenotypic trajectory analyses. Behavioral Ecology and Sociobiology, 68(10), 15771587. doi:10.1007/s00265-014-1767-y

Ingley, S. J., Billman, E. J., Belk, M. C., \& Johnson, J. B. (2014b). Morphological Divergence Driven by Predation Environment within and between Species of Brachyrhaphis Fishes. Plos One, 9(2). doi:10.1371/ journal.pone.0090274

Ingley, S. J., Reina, R. G., Bermingham, E., \& Johnson, J. B. (2015). Phylogenetic analyses provide insights into the historical biogeography and evolution of $\mathrm{Bra}$ chyrhaphis fishes. Molecular Phylogenetics \& Evolution 89, 104-114. doi:http://dx.doi.org/10.1016/j. ympev.2015.04.013.

Ingley, S. J., \& Johnson, J. B. (2016a). Divergent natural selection promotes immigrant inviability at early and late stages of evolutionary divergence. Evolution, 70, 600-616. doi: 10.1111/evo.12872

Ingley, S. J., \& Johnson, J. B. (2016b). Selection is stronger in early-versus-late stages of divergence in a Neotropical livebearing fish. Biology Letters, 12(3). doi:10.1098/rsbl.2015.1022

Johnson, J. B. (2002). Divergent life histories among populations of the fish Brachyrhaphis rhabdophora: detecting putative agents of selection by candidate model analysis. Oikos, 96(1), 82-91. doi:10.1034/j.1600-0706.2002.960109.x

Johnson, J. B., \& Belk, M. C. (2001). Predation environment predicts divergent life-history phenotypes among populations of the livebearing fish Brachyrhaphis rhabdophora. Oecologia, 126(1), 142-149.

Johnson, J. B., \& Zuniga-Vega, J. J. (2009). Differential mortality drives life-history evolution and population dynamics in the fish Brachyrhaphis rhabdophora. Ecology, 90(8), 2243-2252. doi:10.1890/07-1672.1 
Kelley, J. L., \& Magurran, A. E. (2003). Effects of relaxed predation pressure on visual predator recognition in the guppy. Behavioral Ecology and Sociobiology, $54,225-232$.

Kokko, H., \& Rankin, D. J. (2006). Lonely hearts or sex in the city? Density-dependent effects in mating systems. Philosophical Transactions of the Royal Society, 361, 319-334.

Magurran, A. E., Seghers, B. H., Carvalho, G. R., \& Shaw, P. W. (1992). Behavioral consequences of an artificial introduction of guppies (Poecilia reticulata) in $\mathrm{N}$. Trinidad: evidence for the evolution of antipredator behavior in the wild. Proceedings of the Royal Society of London B, 248, 117-122.

Magnhagen, C., Hellstrom, G., Borcherding, J., \& Heynen, M. (2012). Boldness in two perch populations-longterm differences and the effect of predation pressure. Journal of Animal Ecology, 81(6), 1311-1318. doi:10.1111/j.1365-2656.2012.02007.x

Ottoni, E. B. (2000). EthoLog 2.2: a tool for the transcription and timing of behavior observation sessions. Behavior Research Methods, Instruments, \& Computers, 32(3), 446-449.

R Development Core Team (2008). R: A language and environment for statistical computing. $R$ Foundation for Statistical Computing, Vienna, Austria. ISBN 3-900051-07-0. Retrieved from http://www.R-project.org

Reznick, D., \& Endler, J. A. (1982). The impact of predation on life-history evolution in trinidadian guppies (Poecilia reticulata). Evolution, 36(1), 160-177. doi: $10.2307 / 2407978$

Riesch, R., Martin, R. A., \& Langerhans, R. B. (2013). Predation's Role in Life-History Evolution of a Livebearing Fish and a Test of the Trexler-DeAngelis
Model of Maternal Provisioning. American Naturalist, 181(1), 78-93. doi:10.1086/668597

Roff, D. A. (1992). Evolution of life histories: theory and analysis. Springer Science \& Business Media.

Rowe, M., Pierson, K. L., \& McGraw, K. J. (2015). Exploratory behavior is associated with plasma carotenoid accumulation in two congeneric species of waterfowl. Behavioural Processes, 115, 181-190. doi:10.1016/j. beproc.2015.04.008

Schluter, D. (2000). The ecology of adaptive radiation. Oxford University Press.

Seghers, B. H. (1974). Schooling Behavior in the Guppy (Poecilia reticulata): An Evolutionary Response to Predation. Evolution, 28, 486-489.

Simcox, H., Colegrave, N., Heenan, A., Howard, C., \& Braithwaite, V. A. (2005). Context-dependent male mating preferences for unfamiliar females. Animal Behaviour, 70, 1429-1437. doi:10.1016/j. anbehav.2005.04.003

Smith, B. R., \& Blumstein, D. T. (2010). Behavioral types as predictors of survival in Trinidadian guppies (Poecilia reticulata). Behavioral Ecology, 21(5), 919926. doi:10.1093/beheco/arq084

Stamps, J. A. (2007). Growth-mortality tradeoffs and 'personality traits' in animals. Ecology Letters, 10(5), 355-363. doi:10.1111/j.1461-0248.2007.01034.x

Wesner, J. S., Billman, E. J., Meier, A., \& Belk, M. C. (2011). Morphological convergence during pregnancy among predator and nonpredator populations of the livebearing fish Brachyrhaphis rhabdophora (Teleostei: Poeciliidae). Biological Journal of the Linnean Society, 104(2), 386-392. doi:10.1111/j.1095-8312.2011.01715.x 
